Article

\title{
Flexible Patterns for Soft 3D Printed Fabrications
}

\author{
Kanygul Chynybekova ${ }^{1}$ and Soo-Mi Choi ${ }^{1, *(1)}$ \\ Department of Computer Science and Engineering, Sejong University, 209 Neungdong-ro, Gwangjin-gu, \\ Seoul 143-747, Korea; kanyg956@gmail.com \\ * Correspondence: smchoi@sejong.ac.kr
}

Received: 23 October 2019; Accepted: 7 November 2019; Published: 12 November 2019

\begin{abstract}
Rapid improvements in 3D printing technology bring about new possibilities to print with different types of printing materials. New studies have investigated and presented various printing methodologies. However, the majority of these studies are targeted at experimenting with rigid 3D printed objects rather than soft 3D printed fabrications. The presented research considers soft 3D printing, particularly focusing on the development of flexible patterns based on non-homogenous hybrid honeycombs for the interior of 3D printed objects to improve their flexibility and additional stretchability including the lightweight interior. After decomposing the area of an object into regions, our method creates a specific design where patterns are positioned at each partitioned region of the object area by connecting opposite sides of the boundary. The number of regions is determined according to application requirements or by user demands. The current study provides the results of conducted experiments. The aim of this research is to create flexible, stretchable, and lightweight soft 3D printed objects by exploring their deformation responses under tension, compression and flexure tests. This method generates soft 3D printed fabrications with physical properties that meet user demands.
\end{abstract}

Keywords: soft printing; soft 3D printed fabrication; flexible; stretchable; lightweight; soft filament; flexible pattern; non-homogenous hybrid honeycomb structure

\section{Introduction}

Technological innovations in the additive manufacturing field enable us to fabricate different designs with an increasing range of materials. Recently, different communities have expressed a lot of interest in soft 3D printing since the soft filament material type broadens the range of flexible 3D printed objects. Different manufacturers have introduced materials with a variety of flexibility levels, mechanical performance, and qualities to the market. However, these materials do pose some challenges. The soft filament handling process can be different from that of hard filament material. Furthermore, most 3D printers are specifically hard filament type. Despite the adaptation of a soft printing mode, the quality of soft 3D printed objects is usually lower compared to similar rigid 3D prints. Furthermore, most studies focus on rigid 3D fabrications [1-8]. In this research, we closely examine soft 3D printing materials and created a comparison experiment to showcase our developed patterns.

The most important choice for successful soft 3D printed fabrications is the selection of a suitable soft filament. This choice depends on the quality requirements of the project since the quality impacts the level of flexibility of the produced 3D objects. The major advantage of soft-filament materials is the flexibility that makes them deform under a load and revert back to their original state when the load is removed. This property makes it possible to fabricate durable 3D objects with high deformation stability. The hardness scale of soft 3D printing materials is measured with Shore values. For thermoplastic polyurethane (TPU) [9], the hardness scale ranges between Shore A 60-90 and whose level of flexibility can be classified from ultra-flexible to semi-flexible. Different polymer blends are used to create TPU 
and its level of softness depends on this chemical consistency. In addition to its softness and flexibility, TPU is also known for its functional properties of being durable and being able to withstand ambient temperatures up to $80^{\circ} \mathrm{C}$. TPU is therefore practical for both consumer and industrial use. Another soft filament on the market is thermoplastic elastomer (TPE) [10], which was introduced earlier than TPU. It is an elastic printing material that is highly stretchable. It can be stretched to more than twice its original length and return to its original state without permanent deformation. Its elastic properties enable the fabrication of flexible 3D objects. TPE's typical Shore hardness of 85A is softer than that of TPU. The technical characteristics of TPU describe it as much stiffer than TPE. However, both are widely used for soft 3D printed objects that require flexibility and stretchability. Another soft printing material is soft polylactic acid (soft PLA) [11], which is a thermoplastic polymer that is considered to be more eco-friendly compared to other 3D printing materials. The softness of soft PLA is mostly indicated with Shore values. Higher Shore values are used to create less flexible 3D fabrications, while lower Shore values are recommended for printing soft and flexible 3D fabrications. It is important to find a balance between flexibility and printability when working with soft 3D printing materials. Selection of materials requires a careful examination of their technical characteristics as well as a consideration of the application's demands for soft 3D printed fabrications.

In this study, we present honeycomb patterns that are positioned according to decomposed regions in order to create a specific design, which is defined by our method. The number of regions depends on the required demands of the application. The current study included the development of two types of honeycomb patterns with slight differences in their topology. These patterns were also compared to the grid pattern that is currently the standard for infill in 3D printed fabrications. The experiments focused on TPU and TPE printed 3D fabrications. These were compared through a careful examination of their performance under compression, tension and flexure tests that focused particularly on their flexibility, additionally stretchability and bending as well as by looking at their weight to judge the efficiency of material usage. The results of the conducted experiments show that the physical properties of soft 3D printed fabrications are affected by pattern type along with the elasticity of the printing material.

The contribution of our study is as follows:

1. We compared soft printing materials to check criteria that we specified in the experiment.

2. We developed a specific design for the interior of soft 3D printed fabrications along with flexible patterns based on the non-homogenous hybrid honeycomb structure.

3. We conducted experimental compression, tension and flexure tests to reveal the efficiency of each presented pattern.

This paper is organized as follows: Section 2 is dedicated to reviewing works related to soft 3D printing materials and research related to infill patterns. Section 3 lays out the methodology of our interior design along with pattern descriptions. Section 4 describes the flexibility of the proposed honeycomb pattern. Section 5 provides the description of the hardness scale of soft 3D printing materials. Section 6 summarizes the conducted tests to reveal the experimental performance of the proposed patterns with different soft printing materials. Section 7 gives the conclusion.

\section{Related Work}

\subsection{Soft Materials}

Soft 3D printing materials have a variety of features that make them a great choice for a wide range of uses in different fields depending on the chemical consistency, which impacts their properties. As we mentioned earlier, current existing soft 3D printing materials are mostly referred to as TPE and TPU, which are made of different thermoplastic blends. The chemical consistency of these blends plays an important role in the materials' level of flexibility. There are different types of blends with various chemical consistencies that can be grouped into the four major categories: soft, medium soft, medium hard, and hard materials. These range from Shore hardness 20A to 100A. In the past few years, flexible 
filaments been widely used for soft 3D printing. A great number of flexible filament products have been introduced to the market by different manufacturers. One of the most popular flexible filament series is NinjaFlex [12] from the manufacturer, NinjaTek. NinjaFlex has two major lines of TPU and TPE variants that come in a wide range of colors. According to the technical characteristics of NinjaFlex products, its tensile strength is $26 \mathrm{MPa}$, its elongation at break is $660 \%$, and its melting temperature reaches 216 degrees. It claims to be the strongest and most flexible filament on the market, and it is available in $1.75 \mathrm{~mm}$ and $3.00 \mathrm{~mm}$ diameters. There are also a variety of brands of flexible filament products by various manufacturers such as SAINSMART [13], Fillamentum [14], and MadeSolid [15] competing in the market. These are also offered in a wide selection of colors and they come in $1.75 \mathrm{~mm}$ and $2.85 \mathrm{~mm}$ diameters. To fabricate 3D objects with certain technical characteristics and elastic properties, it is necessary to select an appropriate brand of flexible filament products along with a feasible level of flexibility. In study [16], samples were manufactured from NinjaFlex TPU to investigate TPU's material properties and the compressive behavior of 3D printed honeycombs with graded densities. Similarly, researchers carried out another experiment in study [17], where they closely examined the mechanical properties of thick honeycomb structures. Soft 3D printing materials are integrated in many fields, including robotics, and study [18] used robotic fingers printed from soft 3D printing material, where the soft grippers handled objects more gently than rigid robotic fingers due to the elastic properties of their soft material. Another study, [19], experimented with foam structuring for 3D fabrications with controllable elasticity. This method integrates Voronoi foam to generate microstructures with controlled isotropic elastic behavior. The study also provides detailed descriptions for deriving the parameters of structures to produce gradients of elasticity. Their method produces rigid and flexible 3D fabrications through powder sintering 3D printing technology. Study [20] introduced a method with controlled elasticity along different directions, which is an orthotropic k-nearest foam design method that enables the fabrication of 3D objects while considering an input stress field.

There are a limited number of studies dedicated to soft 3D printing, particularly the designing of flexible infill patterns to improve the physical properties of soft 3D printed fabrications. This work provides an early attempt towards exploring soft 3D printed fabrications by presenting a specific interior design that is filled with newly developed flexible infill patterns. The study also explores how soft 3D printed fabrications are affected by both the elastic properties of their materials and their interior design, including the infill pattern type. The experiments utilized two types of 3D printing material, TPU and TPE, to examine their elastic properties as well as their effects on soft 3D printed fabrications and to reveal their compression, tensile and flexure strengths.

\subsection{Infill Patterns}

In 3D printing, structures that are printed in the interior of 3D fabrications are known as infill patterns. These are generated according to designed geometric shapes. Various geometries of infill patterns are offered by slicing tools [21-23], along with infill settings for providing thickness, scale, and printing speed. Unlike the existing slicing tools used in our previous study, [24], this study creates more convenience for users by including an option to use a scaling parameter to specify the number of rows and columns in infill patterns. This makes for a more precise structuring of infill patterns. This study also reviews designs from related studies and classifies them into several categories such as lightweight, lattice patterns, and adaptive infill patterns.

Lightweight patterns: One of the earlier efforts towards creating lightweight designs for the interior of 3D fabrications occurred in study [25]. Researchers used skin frame structures similar to frame structures in architecture. Their method shows an efficiency in the reduction of material usage. Study [26] proposed a method that significantly reduces the weight of 3D fabrications and improves their physical properties by strengthening structurally weak parts of the models. This approach employs a Voronoi diagram to create honeycomb-like infill patterns. Study [27] has been presented as a cost-effective method that reduces 3D printing materials. This study introduced an adaptive voids algorithm for hollowing $3 \mathrm{D}$ fabrications. Their approach generates given a volume 
boundary, a parameterised adaptive infill primal and dual cellular structure for additive manufacturing. Furthermore, in study [28], researchers experimented with a new type of infills named Gyroid structures. Gyroid structures are experimentally verified to be a lightweight design for the interior of $3 \mathrm{D}$ fabrications that also improve the physical properties of the fabrications.

Lattice patterns: Study [29] presented a method that generates lattice structures for 3D fabrications. This study considered several types of lattice structures that can automatically be generated within the input model after the Boolean operations. Study [30] introduced a method for designing lattice structures with a pipeline. This pipeline includes options to modify structure dimensions, strut shape, size, and additional connections between struts. This has been done in order to meet the required user demands for 3D fabrications. In the study [31] developed a method for optimizing lattice structures by utilizing the traditional bidirectional evolutionary structural optimization algorithm. Study [32] proposed a voxel-based method which utilizes voxels to generate different types of lattice structures that can be observed in their resulted 3D fabrications.

Adaptive infill patterns: It is an undeniable fact that denser infill patterns supply stronger support to a fabrication. However, they consume more printing time. Study [33] proposed a new method of optimization based on a quadtree algorithm. This approach optimizes infill patterns to ensure that $3 \mathrm{D}$ fabrications meet the required user demands with less material consumption compared to conventional patterns. This study has similarity to study [34]; both of these studies aim to strengthen the structural weak parts of objects and consume less printing material. Along with these methods, which proposed adaptive infill patterns, researchers in study [35] experimented with adaptive rhombic infill patterns. These patterns strengthen 3D fabrications by applying denser infill patterns to structural weak points.

The printability of infill patterns is extremely important. The printing technology depends on the design of infill patterns and printing material as well as application requirements. For soft 3D printing, there are 3D printing technologies such as the Fused Deposition Method (FDM) [36] and Liquid Deposition Modelling (LDM) [37]. LDM is a relatively new 3D printing technology that was introduced in 2015. It is considered as one of the promising 3D printing technology that fabricates objects with ceramic, wood-based materials, cement, clay and liquid silicone. However, LDM 3D printers are more expensive compared to FDM 3D printers and it requires special handling. FDM 3D printers are popular among users due to its easy handling mode-besides, it can print with rigid and soft materials-in addition there are a variety of affordable FDM 3D printers. Thus, we target to develop flexible infill patterns that are printable with FDM 3D printers, which are the most common and cost-effective.

The reviewed works mostly target rigid 3D fabrications rather than soft 3D printed objects. In our research we developed a specific interior design that positions flexible patterns column by column within each predetermined region in order to improve physical properties of 3D fabrications, such as flexibility and stretchability, while also considering the need to minimize material consumption to reduce cost.

\section{Method}

In this section, we provide the detailed description of our method, precisely the construction of the proposed interior design that positions infill patterns in a specific way by using the pattern columns. The beginning part briefly describes the pattern construction and the remaining part provides the description of the proposed method.

Before we go further, the discussions about homogenous and non-homogenous hybrid honeycomb structures are required, since the flexible pattern is derived from non-homogenous hybrid honeycomb structures. As it is known, homogenous honeycomb structures could be found in nature while non-homogenous hybrid honeycombs are artificially designed for different applications. We are considering non-homogenous hybrid honeycombs which are made of hexagons and rhombs. There are noticeable differences between homogenous and non-homogenous hybrid honeycomb structures 
in their design and mechanical performances because of their topological differences. The differences in their design can be visually observed from Figure 1.

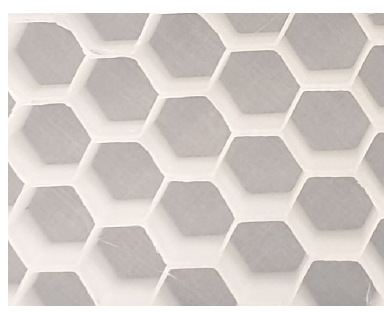

(a)

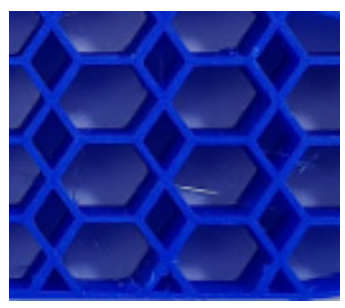

(b)

Figure 1. (a) Homogenous honeycomb structure (b) our non-homogenous honeycomb structure.

The main advantage of non-homogenous hybrid honeycomb structures is that they are lighter than the above shown homogenous honeycombs, which can be noticed from their geometry. The non-homogenous hybrid honeycomb structure consumes less printing material because it consists of a combination of hexagons and rhombs while the homogenous structure comprises only hexagons, the perimeter of the homogenous structures is larger compared to the perimeter of the non-homogenous hybrid honeycomb structure, which can be seen from their design in Figure 1. The proposed method can create vertically and horizontally positioned patterns using the non-homogenous hybrid honeycomb structure.

The method in the current study was developed to generate soft 3D printed fabrications with highly deformable stability. This uses a combination of tailored 3D fabrication with such physical properties as flexibility and supplementary stretchability, while providing a lightweight interior. This goal was accomplished by developing flexible infill patterns based on non-homogenous hybrid honeycomb structures. The developed patterns are applied to the interior of soft $3 \mathrm{D}$ printed fabrications. The flexible honeycomb pattern is constructed according to the designed scheme and its topological part is shown in Figure 2.

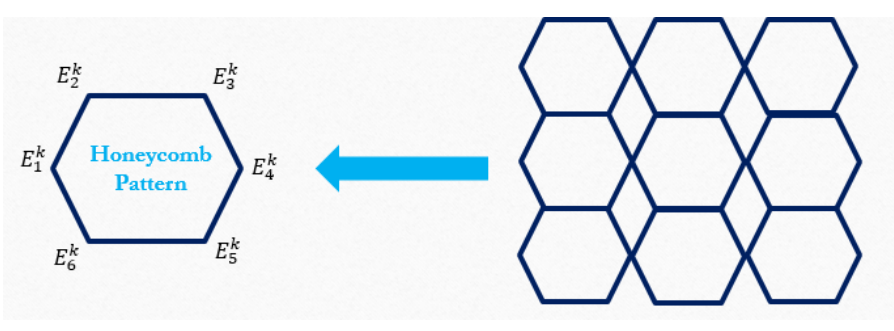

Figure 2. Topology of the honeycomb pattern.

The pattern elements $\forall$ Elements $_{i}^{k-1}$, where $i=Z$ are generated according with the following subdivision matrix:

$$
S_{m}{ }^{k}=\frac{1}{8}\left[\begin{array}{cccc}
6 & 0 & 0 & 2 \\
4 & 4 & 0 & 0 \\
0 & 6 & 2 & 0 \\
0 & 2 & 6 & 0 \\
0 & 0 & 4 & 4 \\
2 & 0 & 0 & 6
\end{array}\right] .
$$

The honeycomb patterns are derived from a symmetric grid mesh Grid $^{k-1}$ with the set of elements Elements ${ }_{i}^{k-1}$, which consists from the set of points $V_{i}$ where $i=Z$, it can be written in the following form as Elements ${ }_{i}^{k-1}=\left[V_{1}, V_{2}, \ldots, V_{n}\right]$ where $\mathrm{n}=\mathrm{Z}$, Elements ${ }_{i}^{k-1} \in$ Grid $^{k-1}$ and $\forall V$ can be written as $V=\left[E_{1}^{k-1}, E_{2}^{k-1}, E_{3}^{k-1}, E_{4}^{k-1}\right], V \in G r i d^{k-1}$.

$$
V=V^{T}
$$




$$
E_{i}^{k}=S_{m}^{k} V,
$$

where $S_{m}{ }^{k}$ is the subdivision matrix and $E_{i}^{k}$ is a new set of points.

In the above, we described the pattern construction along with the topological part. Further, we focused on the presented method. With our approach, the interior of a 3D fabrication is decomposed into different regions, where the number of regions depends on the application requirements or the user's demands.

$$
\text { Method }=I / P,
$$

where $I$ is the interior area of the object and $P$ is the number of partitioned regions. Within the region, a certain interval created a column of pattern elements.

The proposed method was built with accordance of the following block diagram that can be seen from Figure 3. The diagram shows major stages for processing input models in order to produce objects with the presented interior design and infill patterns.

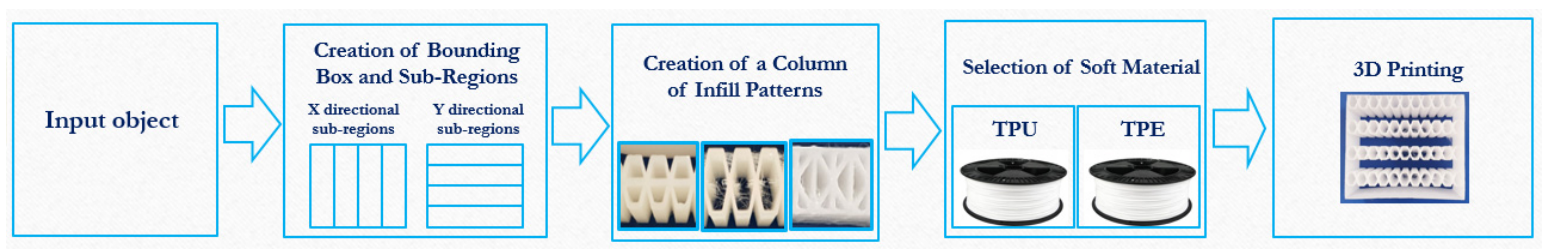

Figure 3. The diagram for the proposed method.

For each sub-region, the Euclidean distance for each region is calculated as follows:

$$
E D^{2}=\left(x_{n+1}-x_{n}\right)^{2}+\left(y_{n+1}-y_{n}\right)^{2}+\left(z_{n+1}-z_{n}\right)^{2}
$$

The regions are symmetric or asymmetric depending on the application requirements and they are equidistant from each other as: $\forall E D\left(h_{n}, h_{n+1}\right)=E D\left(h_{1}, h_{2}\right)$, where $h=\left[h_{1}, \ldots . h_{n+1}\right]$ are points.

A column of pattern elements is created for each partitioned region and the presented honeycomb pattern can be position in biaxial directions, as shown in Figure 4. There are four regions where we created columns of flexible honeycomb patterns. Each column intervals are specified in Table 1.

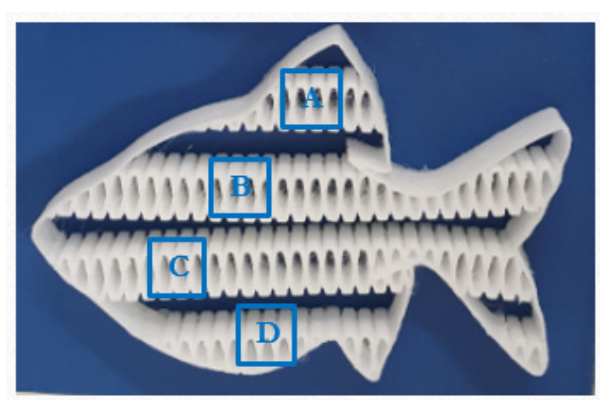

(a)

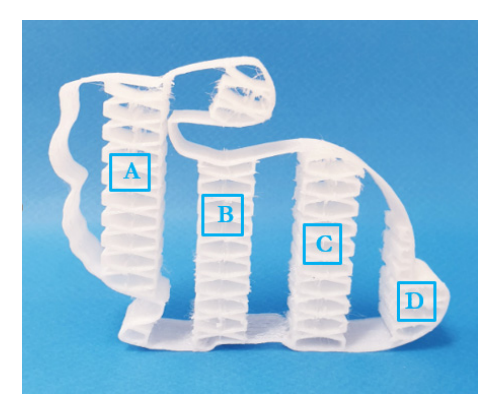

(b)

Figure 4. Creation of patterns for each partitioned region, (a) the size of the fish model is $14 \mathrm{~cm} \times 8 \mathrm{~cm}$ $\times 2 \mathrm{~cm}$, (b) the size of the bunny model is $10 \mathrm{~cm} \times 8 \mathrm{~cm} \times 2 \mathrm{~cm}$.

Table 1. Column intervals.

\begin{tabular}{cccc}
\hline $\boldsymbol{A}$ & $\boldsymbol{B}$ & $\boldsymbol{C}$ & $\boldsymbol{D}$ \\
\hline$A_{1} \leq x \leq A_{n}$ & $B_{1} \leq x \leq B_{n}$ & $C_{1} \leq x \leq C_{n}$ & $D_{1} \leq x \leq D_{n}$ \\
or & or & or & or \\
$A_{1} \leq y \leq A_{n}$ & $B_{1} \leq y \leq B_{n}$ & $C_{1} \leq y \leq C_{n}$ & $D_{1} \leq y \leq D_{n}$ \\
Column $_{1} \in\left[A_{n-1}, A_{n}\right]$ & Column $_{2} \in\left[B_{n-1}, B_{n}\right]$ & Column $_{3} \in\left[C_{n-1}, C_{n}\right]$ & Column $_{4} \in\left[D_{n-1}, D_{n}\right]$ \\
\hline
\end{tabular}


In this study, we introduced two types of honeycomb patterns: (1) a regular honeycomb pattern and (2) a honeycomb trapezoid with a slightly distinctive topology, as shown in Figure 5. For the comparison test we have investigated a standard grid-type pattern.

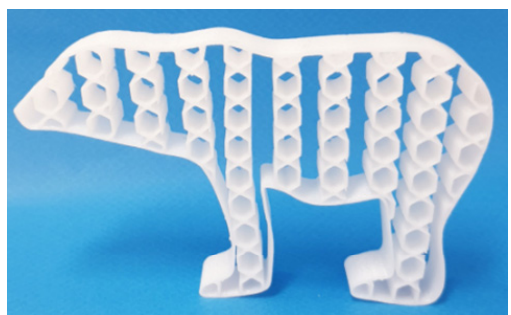

(a)

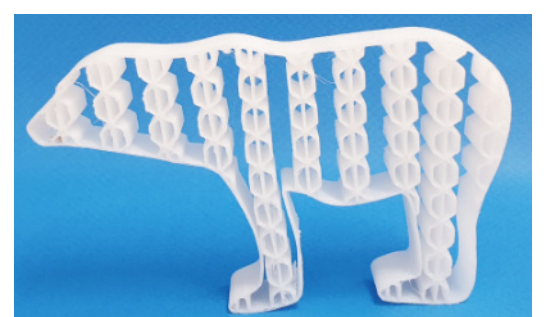

(b)

Figure 5. (a) Honeycomb pattern and (b) hexagonal trapezoid pattern. The size of the bear models is $14 \mathrm{~cm} \times 8 \mathrm{~cm} \times 2 \mathrm{~cm}$.

\section{Flexibility of the Honeycomb Pattern}

The mechanical properties of honeycomb patterns can be described by analyzing a single honeycomb pattern element. Furthermore, it is necessary to take into consideration its geometrical parameters, since the parameters will change when the element is subjected to compression loads, as was explored in a similar study [38]. The pattern element along with its geometric parameters are shown in Figure 6.

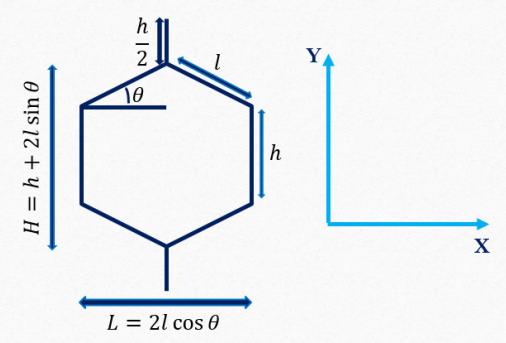

Figure 6. Geometric parameters of the honeycomb pattern element.

We denoted the parameters of the element as follows: $h$ is the height of the side, $l$ is the length of the side, where $\theta$ is the angle between the horizontal and inclined edges that we consider as $\theta=30^{\circ}$ because we are considering a regular hexagonal element. The hexagonal element's overall width is defined as $L=2 l \cos \theta$ and the overall height is $H=h+2 l \sin \theta$ In the current study, it is important to note that $\mathrm{H}$ is defined as the full overall height of the element, while in other similar studies-as in the study [39], based on the homogenous honeycomb structure, used half of the overall height of the honeycomb cell. In our case, the element is fully affected by compression, tension- and flexure loads since the presented flexible pattern is derived from non-homogenous honeycomb. Depending on the strength of the loads, the geometric parameters of the honeycomb pattern will be changed and will be different than its original parameters.

To test the flexibility of the honeycomb pattern, we conducted a simple compression test, which can be observed in Figure 7. Figure 7 shows how infill patterns are affected when we apply a y-directional compression load with a pen, which was used for visual observation of the pattern flexibility. The strength of the applied load changes the geometric parameters of the element-hence it becomes $L \neq 2 l \cos \theta$ and $H \neq h+2 l \sin \theta$ during the compression. There are affected areas of the element along the $x$ and $y$ axial direction is defined as follows:

$$
A_{x}=(2 l \cos \theta) b \quad A_{y}=(h+2 l \sin \theta) b,
$$


where $A_{x}$ and $A_{y}$ are the affected areas of the element by compression load, $\mathrm{b}$ is the depth of the element;

To provide detailed insights for the honeycomb deformation mechanisms we provided a model for biaxial loads, but we practically considered y-directional load in Figure 7. The element sides are considered as beams. As it is known, the bending moment is the reaction induced in a honeycomb element, which tends to bend the walls of the pattern element when the load is applied. We defined the moment $M$ on the $x$ and $y$ directional beam as follows:

$$
M_{x}=\frac{P_{x} l \sin \theta}{2} \text { and } M_{y}=\frac{P_{y} l \cos \theta}{2},
$$

where $P_{x}$ and $P_{y}$ are the axial loads; $M_{x}$ and $M_{y}$ are the bending moments on the $x$ and $y$ directional beam;

The loads can be defined from the following equation:

$$
P_{x}=\sigma_{1}(h+2 l \sin \theta) b \text { and } P_{y}=\sigma_{2}(2 l \cos \theta) b,
$$

where $\sigma_{1}$ and $\sigma_{2}$ are $\mathrm{x}$ and $\mathrm{y}$ directional forces;

Here, in the above-mentioned Equation (7), we considered the directional overall height $H$ and the overall length $L$.

As it is shown in Figure 7, the pattern elements bend easily because of the elastic properties of the soft 3D printing materials, TPU and TPE, and due to the geometry of the honeycomb patterns.

When the load is removed, the pattern elements return to their original state without permanent deformation due to the flexibility of the patterns and the material's elasticity.

In Figure 7, the bunny models with larger and medium size patterns can be seen, the models were tested by a pen and metalic stick to visually examine their flexibility. Furthermore, to provide a quantified evidence of the flexibility, the bunny model was tested by the UTM machine INSTRON-5690 (USA, INSTRON). The result showed that its compression strength is more than $1000 \mathrm{~N}$.

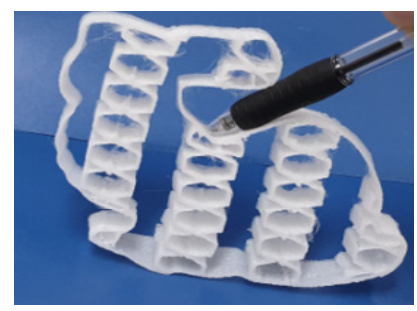

(a)

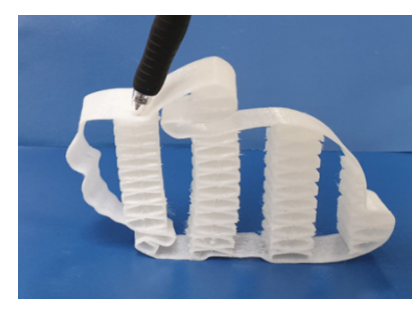

(b)

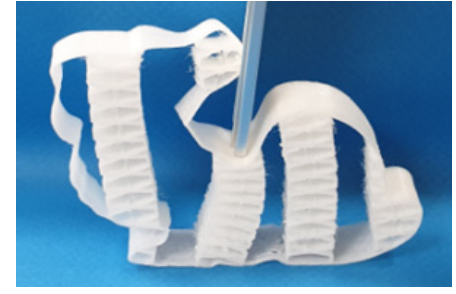

(c)

Figure 7. Compression effects on bunny model, the size of bunny models is $10 \mathrm{~cm} \times 8 \mathrm{~cm} \times 2 \mathrm{~cm}$. (a) the bunny model with larger infills tested with a pen, (b) the bunny model with smaller infills tested with a pen (c) the bunny model with smaller infills tested with metallic stick.

In the current study, the built model that determines the flexibility of the honeycomb pattern is different from the majority of the proposed models. Our flexible honeycomb patterns are derived from the non-homogenous honeycombs, and for this reason we considered the full overall height in Young's moduli as follows:

$$
\begin{gathered}
E_{x}=\frac{F_{x} / A_{x}}{U_{x} / L_{x}}=\frac{F_{x} L_{x}}{A_{x} U_{x}} \text { and } E_{y}=\frac{F_{y} / A_{y}}{U_{y} / L_{y}}=\frac{F_{y} L_{y}}{A_{y} U_{y}}, \\
L_{x}=L \text { and } L_{y}=H \\
L=2 l \cos \theta \text { and } H=h+2 l \sin \theta
\end{gathered}
$$

where $E_{x}$ and $E_{y}$ are the Young moduli in $x$ and $y$ axial directions; $F_{x}$ and $F_{y}$ is the applied forces in $\mathrm{x}$ and $\mathrm{y}$ directions that needed to produce a unit displacement; $L_{x}$ is the width of the element along $\mathrm{x}$-direction and $L_{y}$ is height of the element along y-direction; $A_{x}$ and $A_{y}$ are the areas of the element along the $\mathrm{x}$ and $\mathrm{y}$ directions. $U_{x}$ and are the displacements in $\mathrm{x}$ and $\mathrm{y}$ directions. 
Another elastic component, such as $v_{x y}, v_{y x}$, is the Poisons ratio $\mathrm{x}$, and $\mathrm{y}$ axial directions can be defined from the following equation:

$$
v_{x y}=-\frac{\varepsilon_{y}}{\varepsilon_{x}} \text { and } v_{y x}=-\frac{\varepsilon_{x}}{\varepsilon_{y}}
$$

where $\varepsilon_{x}, \varepsilon_{y}$ are $\mathrm{x}$ and $\mathrm{y}$ axial directional strains.

The shear modulus $G_{x y}$ is defined as follows:

$$
G_{x y}=\frac{\text { Shear } \text { stress }_{x y}}{\text { Shear } \operatorname{strain}_{x y}}
$$

We can conclude that the proposed interior design and the geometry of the patterns, as well as material properties, make our 3D fabrications more flexible. The proposed interior design combines flexibility and low weight, which are valuable physical properties for 3D fabrications. Additionally, we examined the flexibility of samples fully filled with the homogenous honeycomb structures and sparse homogenous honeycombs, as can be seen in Figure 8.

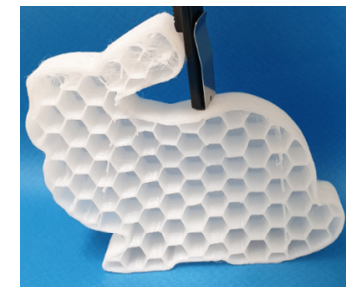

(a)

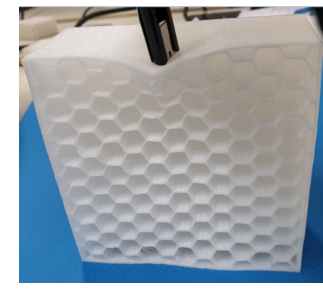

(b)

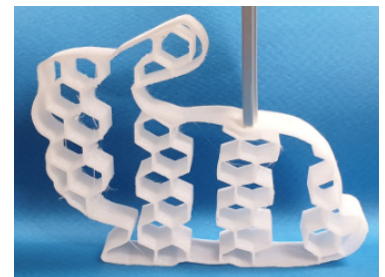

(c)

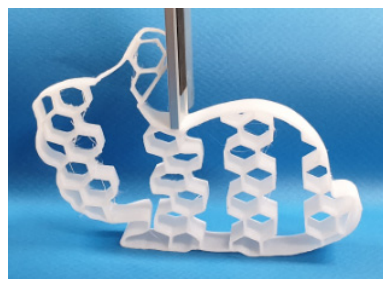

(d)

Figure 8. Compression effects on the models filled with the homogenous honeycomb structure, the size of bunny models is $10 \mathrm{~cm} \times 8 \mathrm{~cm} \times 2 \mathrm{~cm}$, the size of the cube model is $10 \mathrm{~cm} \times 10 \mathrm{~cm} \times 5 \mathrm{~cm}$, (a) the bunny model with fully filled homogenous honeycomb, it is tested with a metallic stick, (b) the box model filled with homogenous honeycomb, its backside is tested with a metallic stick, (c) the bunny model with sparse homogenous honeycomb, its back side is tested with a metallic stick, (d) the bunny model with sparse homogenous honeycomb, its front side is tested with a metallic stick.

As depicted in Figure 8, we applied y-directional compression load with a metallic stick, which is heavier than a pen to provide more sufficient compression load for the samples that are fully filled with the homogenous honeycomb structures. Despite the elastic properties of soft 3D printing materials, a very slight bending effect was observed at the top of models. Here, homogenous honeycomb structures are non-orthotropic. The compression strength of the bunny model was only $40 \mathrm{~N}$, which backs up the above statement. It is less flexible compared to the bunny model with our proposed interior design and the patterns.

Further, we have experimented with samples that are filled with the sparse honeycomb patterns, as shown in Figure 8. Here, the pattern is derived from the homogenous honeycomb structure. To determine the flexibility of the samples we carried out a test where the samples were subjected to $\mathrm{y}$-directional compression load with a metallic stick. The metallic stick was used in order to provide a stronger compression load. We observed that it is still less flexible than our proposed honeycomb pattern. However, it was slightly better compared to the samples with fully filled homogenous honeycomb structures. From the current examination, we can conclude that the interior filled with the homogenous honeycomb structures are less flexible compared to our proposed pattern. Moreover, our patterns can be flexible in two directions because they are based on the non-homogenous honeycomb structures, which enables to position the columns of patterns vertically and horizontally with the entire elements by our method. 


\section{Soft Printing Materials}

Thermoplastic polyurethane (TPU)

The mechanical properties of TPU are dependent on its chemical consistency, as we described earlier. We further focused on the hardness scale. The Shore hardness of TPU is important because it is a measurement that describes TPU properties. Generally, the Shore hardness of TPU begins from A to D. The category A denotes a flexible type of TPU, while the category D refers to more rigid TPU, the relationship between categories and the TPU and TPE hardness range is depicted in Figure 9.

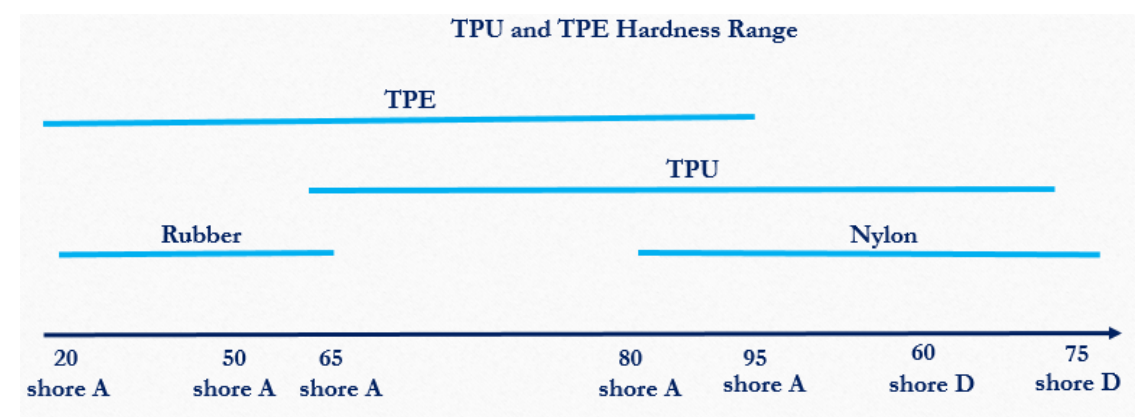

Figure 9. Thermoplastic polyurethane (TPU) categories and its with thermoplastic elastomer (TPE) hardness range.

Thermoplastic elastomer (TPE)

Similarly, with TPU, TPE materials have a variety level of flexibility and softness that is determined by its chemical blends. Because of the elastic properties of TPE users can fabricate soft and flexible 3D fabrications, which are also durable. The printing process can be challenging for TPE compared to TPU because of several factors as the printing speed, temperature and settings. To avoid inaccuracy, it is recommended to set the lower printing speed at 5 to $30 \mathrm{~mm} / \mathrm{s}$.

As mentioned above, the characteristic of soft 3D printing materials is determined by Shore hardness. This hardness scale allows users to fabricate with a required level of flexibility. The level of flexibility can be determined according to the hardness scale table in Table 2.

Table 2. Hardness scale.

\begin{tabular}{cccc}
\hline \multicolumn{4}{c}{ Hardness scale (Shore A) } \\
\hline No. & Category of Objects & Hardness \\
\hline 1. & Shopping cartwheel & $100 \mathrm{~A}$ & Hard \\
2. & Phone cord & $90 \mathrm{~A}$ & Medium Hard \\
3. & Leather belt & $85 \mathrm{~A}$ & Medium Soft \\
4. & Tire tread & $60 \mathrm{~A}$ & Soft \\
5. & Pencil eraser & $40 \mathrm{~A}$ & \\
6. & Rubber band & $20 \mathrm{~A}$ & \\
\hline
\end{tabular}

In this study, TPU printed 3D fabrications with the Shore $80 \mathrm{~A}$ and TPE printed 3D fabrication with the hardness scale is $60 \mathrm{~A}$ were used.

\section{Experimental Results}

As mentioned in the introduction, soft printing materials come in a variety of chemical consistencies and different levels of hardness. Therefore, we printed our outputs with different soft printing materials, such as TPU and TPE, to conduct a comparison test between these outputs to determine the physical properties of 3D fabrications. 
Moreover, we consider how the materials impact the flexibility and additionally stretchability of the soft 3D printed fabrications. The mechanical performance of our outputs was measured under compression using the UTM machine INSTRON-5690 (USA, Instron).

Tension test results

The results for the conducted tension test are provided in the Table 3. We conducted tensile stress tests for different soft printing materials. From the results of our experiment we can conclude that soft $3 \mathrm{D}$ printed fabrications are impacted by factors as the pattern type and the material.

Table 3. Results of tension test.

\begin{tabular}{cccc}
\hline \multirow{2}{*}{ No. } & \multirow{2}{*}{ Tension Strength } \\
\cline { 3 - 4 } & & \multicolumn{2}{c}{ Material } \\
\cline { 3 - 4 } & Box & TPU & TPE \\
\hline \multirow{2}{*}{1} & $10 \mathrm{~cm} \times 5 \mathrm{~cm} \times 1.5 \mathrm{~cm}$ & $778 \mathrm{~N}$ & $1400 \mathrm{~N}$ \\
\hline
\end{tabular}

The tension experiment showed varying results for TPU and TPE printed samples because of their material properties. The most stretchable sample is a 3D print made of TPE. The current experiment verifies the technical characteristics of TPE and TPU regarding their stretchability.

Compression test results

The experimental test results for the compression test are shown in Table 4. The compression test was done to reveal the compression strength of our proposed patterns. When a compression load was applied, the linear elastic deformation is occurred by causing the patterns elements to bend.

Table 4. Results of compression test.

\begin{tabular}{ccccc}
\hline \multirow{2}{*}{ No. } & Model & Material & \multicolumn{2}{c}{ Compression Strength } \\
\cline { 4 - 4 } & & & X-Directional & Y-Directional \\
\hline $\mathbf{1}$ & $\begin{array}{c}\text { Box Model with honeycomb patterns } \\
10 \mathrm{~cm} \times 10 \mathrm{~cm} \times 5 \mathrm{~cm}\end{array}$ & TPU & $503 \mathrm{~N}$ & $122 \mathrm{~N}$ \\
\hline
\end{tabular}

The results of the test revealed that the compressive strength of a pattern directly depends on the elastic properties of its material, as well as on the geometrical shape of its infill pattern. The experiment verified the flexibility of our patterns where loading conditions were conducive to buckling.

Flexural Test

In Table 5 has shown the results of flexure test. As it is known from the physics that flexible materials have lower flexural strengths compared to rigid materials; therefore, we have lower flexural strengths for 3D printed models with TPE and the higher strengths for samples with TPU. Besides, the pattern topology impacts their flexural strengths.

Table 5. Results of flexural test.

\begin{tabular}{cccc}
\hline \multirow{2}{*}{ No. } & Model & \multicolumn{2}{c}{ Flexural Strength } \\
\cline { 3 - 4 } & $\mathbf{1}$ & Honeycomb Pattern & Hexagonal Trapezoid \\
\hline $\mathbf{2}$ & $10 \mathrm{~cm} \times 10 \mathrm{~cm} \times 5 \mathrm{~cm}$ & $212 \mathrm{~N}$ & $343 \mathrm{~N}$ \\
\hline $\begin{array}{c}\text { Box with TPE } \\
10 \mathrm{~cm} \times 10 \mathrm{~cm} \times 5 \mathrm{~cm}\end{array}$ & $75 \mathrm{~N}$ & $216 \mathrm{~N}$ \\
\hline
\end{tabular}


Weight comparison

In addition to determining the optimal pattern for flexibility, we have included a weight comparison of the fabricated 3D objects. This part of the experiment allows us to determine the cost-effectiveness of our method. We measured the weight of each presented model to reveal the lightest interior for soft 3D printed fabrications. These results are shown in Table 6. As it can be seen, there are three different patterns presented with the proposed interior design. The experiment reveals that the lightest model is the fish model with the hexagonal interior, which weighs $47 \mathrm{~g}$. The second lightest is the model with the hexagonal trapezoid interior.

Table 6. Weight of fish models.

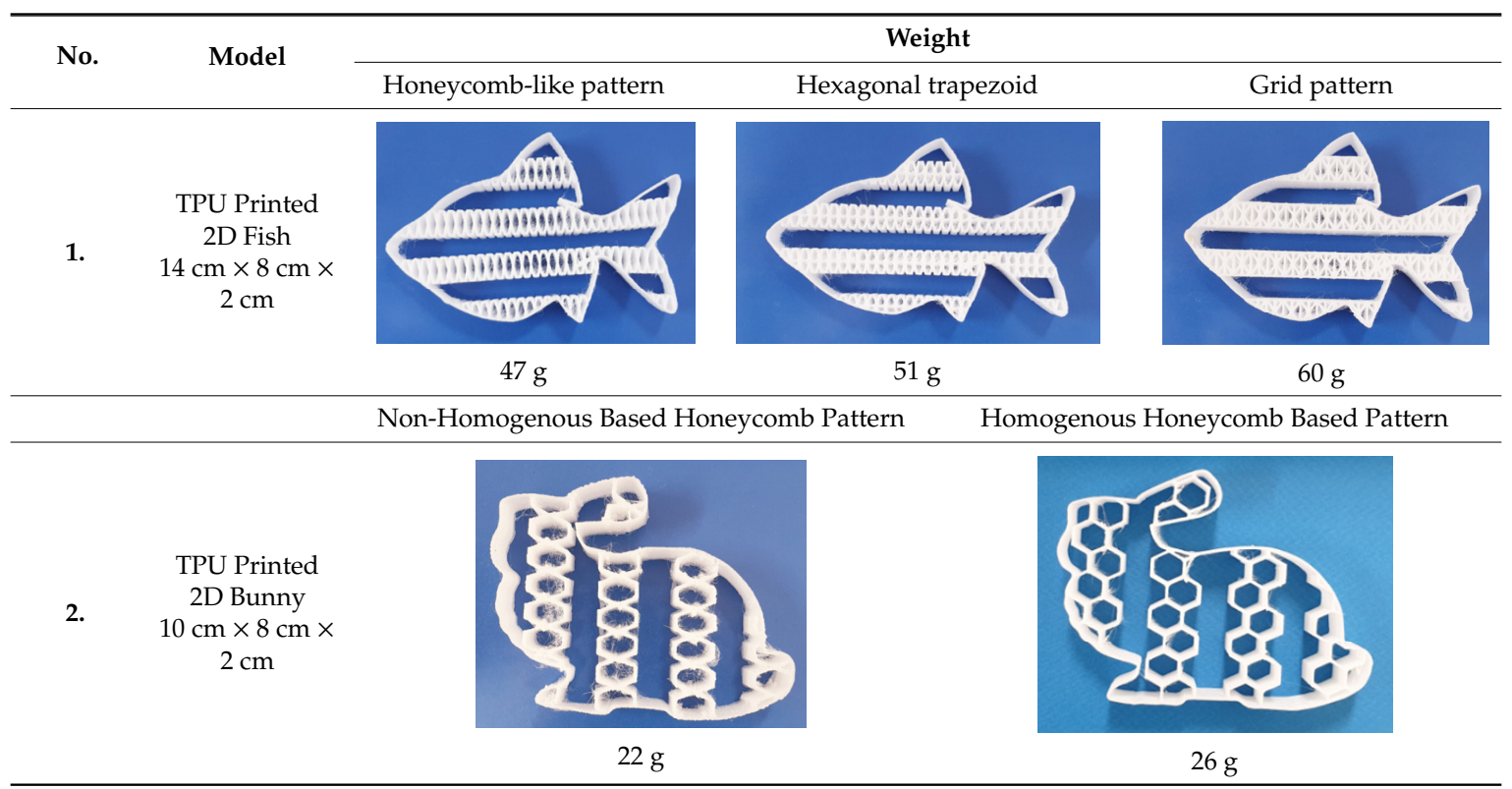

In Table 6, we also tested homogenous and non-homogenous honeycombs regarding their weights. It is shown that our honeycomb pattern is lighter than the homogenous based honeycomb structure.

In Table 7, we compared different sizes of the honeycomb pattern with the standard grid pattern. The lightest soft 3D printed fabrication is the bunny model with the weight of $22 \mathrm{~g}$.

Table 7. Weight comparison of bunny models with different patterns and patterns size.

\begin{tabular}{cccc}
\hline No. & Model & Weight & Grid Pattern \\
\cline { 3 - 5 } & & Honeycomb-like Pattern \\
TPU Printed \\
$2 \mathrm{D}$ Bunny \\
$10 \mathrm{~cm} \times 8 \mathrm{~cm} \times 2 \mathrm{~cm}$
\end{tabular}

In Table 8 we have done a comparison test for 3D models from different categories. The experiment was carried out by measuring their weight one by one. The lightest interior is the hexagonal pattern. Hexagonal patterns are the most efficient patterns regarding their cost-effectiveness and flexibility including stress-sustainability. Therefore, they are widely used in different applications. 
Table 8. Weight comparison of models.

\begin{tabular}{|c|c|c|c|c|}
\hline \multirow{2}{*}{ No. } & \multirow{2}{*}{ Model } & \multirow{2}{*}{ Material } & \multicolumn{2}{|c|}{ Weight } \\
\hline & & & Honeycomb-like Pattern & Hexagonal Trapezoid \\
\hline \multirow[t]{2}{*}{1.} & $\begin{array}{c}\text { 2D Bear } \\
14 \mathrm{~cm} \times 8 \mathrm{~cm} \times 2 \mathrm{~cm}\end{array}$ & TPU & $(2)$ & \\
\hline & & & $34 \mathrm{~g}$ & $41 \mathrm{~g}$ \\
\hline \multirow[t]{2}{*}{2.} & $\begin{array}{c}\text { 3D Box } \\
10 \mathrm{~cm} \times 10 \mathrm{~cm} \times 5 \mathrm{~cm}\end{array}$ & TPU & & \\
\hline & & & $66 \mathrm{~g}$ & $103 \mathrm{~g}$ \\
\hline \multirow[t]{2}{*}{3.} & $\begin{array}{c}\text { 3D Box } \\
10 \mathrm{~cm} \times 10 \mathrm{~cm} \times 5 \mathrm{~cm}\end{array}$ & TPU & 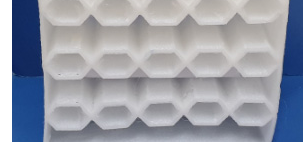 & 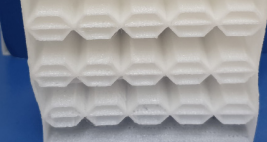 \\
\hline & & & $62 \mathrm{~g}$ & $79 \mathrm{~g}$ \\
\hline \multirow[t]{2}{*}{4.} & $\begin{array}{c}\text { 3D Box } \\
10 \mathrm{~cm} \times 10 \mathrm{~cm} \times 5 \mathrm{~cm}\end{array}$ & TPE & $e_{-\infty}^{-1-}$ & 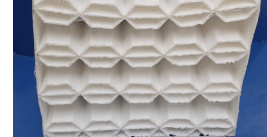 \\
\hline & & & $60 \mathrm{~g}$ & $80 \mathrm{~g}$ \\
\hline
\end{tabular}

\section{Conclusions}

In this study, we have presented a specific interior design targeted for soft 3D printed fabrications along with specially designed flexible infill patterns. It was done to fabricate 3D objects with improved physical properties by focusing on their flexibility and additionally stretchability with consideration for material and cost reduction. Moreover, we described the flexibility of the developed infill patterns and highlighted how the following factors such as the pattern type and the elastic properties of materials affect the physical properties of soft 3D printed fabrications. Through the experiments, we studied the effects of compression, tension and flexure loads on infill patterns. In this paper, we did not consider experiments of overhanging models and models with different thicknesses. In addition, our method is specifically targeted for the FDM 3D printing technology. We recognize these limitations of our study. We fabricated samples by using Flashforge Finder FDM 3D printer (Zhejiang Flashforge3D technology Co., LTD, China). The presented method proved its efficiency in a number of the conducted experiments. From the study investigations, it can be concluded that our method is an effective structural design concept in which materials are properly chosen along with an appropriate pattern type according to their elastic properties. We believe this study brings new innovations into soft 3D printing by providing these meaningful insights into the design process. 
Author Contributions: Conceptualization, K.C.; Funding acquisition, S.-M.C.; Investigation, K.C.; Methodology, K.C.; Project administration, S.-M.C.; Software, K.C.; Supervision, S.-M.C.; Validation, S.-M.C.; Visualization, K.C.; Writing—original draft, K.C.; Writing—review \& editing, K.C. and S.-M.C.

Funding: This research was funded by Ministry of Science and ICT, Korea: IITP-2019-2016-0-00312.

Conflicts of Interest: The authors declare no conflict of interest.

\section{References}

1. Liu, X.; Lin, L.; Wu, J.; Wang, W.; Yin, B.; Wang, C.C.L. Generating sparse self-supporting wireframe models for 3D printing using mesh simplification. Graph. Models 2018, 98, 14-23. [CrossRef]

2. Lee, M.; Fang, Q.; Cho, Y.; Ryu, J.; Liu, L.; Kim, D.S. Support-free hollowing for 3D printing via Voronoi diagram of ellipses. Comput. Aided Des. 2018, 101, 23-36. [CrossRef]

3. Hu, J.; Wang, S.; Wang, Y.; Li, F.; Luo, Z. A lightweight methodology of 3D printed objects utilizing multi-scale porous structures. Vis. Comput. 2019. [CrossRef]

4. Feng, J.; Fu, J.; Lin, Z.; Shang, C.; Li, B. A review of the design methods of complex topology structures for 3D printing. Vis. Comput. Ind. Biomed. Art 2018, 1. [CrossRef]

5. Li, D.; Dai, N.; Zhou, X.; Huang, R.; Liao, W. Self-supporting interior structures modeling for buoyancy optimization of computational fabrication. Int. J. Adv. Manuf. Technol. 2017, 95, 825-834. [CrossRef]

6. Wadbro, E.; Niu, B. Multiscale design for additive manufactured structures with solid coating and periodic infill pattern. Comput. Methods Appl. Mech. Eng. 2019, 357, 112605. [CrossRef]

7. Wei, X.R.; Zhang, Y.H.; Geng, G.H. No-infill 3D Printing. 3D Res. 2016, 7. [CrossRef]

8. Moradi, M.; Meiabadi, S.; Kaplan, A. 3D Printed Parts with Honeycomb Internal Pattern by Fused Deposition Modelling; Experimental Characterization and Production Optimization. Met. Mater. Int. 2019. [CrossRef]

9. Kim, J.H.; Kim, G.H. Effect of rubber content on abrasion resistance and tensile properties of thermoplastic polyurethane (TPU)/rubber blends. Macromol. Res. 2014, 22, 523-527. [CrossRef]

10. Grady, B.P.; Cooper, S.L.; Robertson, C.G. Thermoplastic Elastomers. In The Science and Technology of Rubber; Academic Press: Cambridge, MA, USA, 2013; pp. 591-652.

11. Awale, R.; Ali, F.; Azmi, A.; Puad, N.; Anuar, H.; Hassan, A. Enhanced Flexibility of Biodegradable Polylactic Acid/Starch Blends Using Epoxidized Palm Oil as Plasticizer. Polymers 2018, 10, 977. [CrossRef] [PubMed]

12. Available online: https://ninjatek.com (accessed on 11 November 2019).

13. Available online: https://www.sainsmart.com (accessed on 11 November 2019).

14. Available online: https://fillamentum.com (accessed on 11 November 2019).

15. Available online: https://www.facebook.com/MadeSolid (accessed on 11 November 2019).

16. Bates, S.R.; Farrow, I.R.; Trask, R.S. Compressive behaviour of 3D printed thermoplastic polyurethane honeycombs with graded densities. Mater. Des. 2018. [CrossRef]

17. Hedayati, R.; Sadighi, M.; Mohammadi Aghdam, M.; Zadpoor, A. Mechanical Properties of Additively Manufactured Thick Honeycombs. Materials 2016, 9, 613. [CrossRef] [PubMed]

18. Amiri Moghadam, A.A.; Alaie, S.; Deb Nath, S.; Aghasizade Shaarbaf, M.; Min, J.K.; Dunham, S.; Mosadegh, B. Laser Cutting as a Rapid Method for Fabricating Thin Soft Pneumatic Actuators and Robots. Soft Robot. 2018, 5, 443-451. [CrossRef] [PubMed]

19. Martínez, J.; Dumas, J.; Lefebvre, S. Procedural voronoi foams for additive manufacturing. ACM Trans. Graph. 2016, 35, 1-12. [CrossRef]

20. Martínez, J.; Song, H.; Dumas, J.; Lefebvre, S. Orthotropic k-nearest foams for additive manufacturing. ACM Trans. Graph. 2017, 36, 121. [CrossRef]

21. Available online: https://ultimaker.com/en/products/ultimaker-cura-software (accessed on 11 November 2019).

22. Available online: http://www.kisslicer.com/ (accessed on 11 November 2019).

23. Available online: https://slic3r.org/ (accessed on 11 November 2019).

24. Chynybekova, K.; Choi, S.M. Multilevel Design for the Interior of 3D Fabrications. Symmetry 2019, 11, 1029. [CrossRef]

25. Wang, W.; Wang, T.Y.; Yang, Z.; Liu, L.; Tong, X.; Tong, W.; Deng, J.; Chen, F.; Liu, X. Cost-effective printing of 3D objects with skin-frame structures. ACM Trans. Graph. 2013, 32, 177:1-177:10. [CrossRef]

26. Lu, L.; Sharf, A.; Zhao, H.; Wei, Y.; Fan, Q.; Chen, X.; Savoye, Y.; Tu, C.; Cohen-Or, D.; Chen, B. Build-to-last: Strength to weight 3D printed objects. ACM Trans. Graph. 2014, 33, 97:1-97:10. [CrossRef] 
27. Sa, A.M.; Mello, V.M.; Rodriguez Echavarria, K.; Covill, D. Adaptive Voids Primal and Dual Adaptive Cellular Structures for Additive Manufacturing. Vis. Comput. 2015, 31, 799-808.

28. Li, D.; Dai, N.; Jiang, X.; Jiang, X.; Chen, X. Interior structural optimization based on the density-variable shape modeling of 3D printed objects. Int. J. Adv. Manuf. Technol. 2016, 83, 1627-1635. [CrossRef]

29. Nguyen, D.S.; Vignat, F. A method to generate lattice structure for Additive Manufacturing. In Proceedings of the 2016 IEEE International Conference on Industrial Engineering Engineering Management (IEEM), Bali, Indonesia, 4-7 December 2016. [CrossRef]

30. Chen, Y. A Mesh-Based Geometric Modeling Method for General Structures. In Proceedings of the 26th Computers and Information in Engineering Conference, Philadelphia, PA, USA, 10-13 September 2006. [CrossRef]

31. Tang, Y.; Kurtz, A.; Zhao, Y.F. Bidirectional Evolutionary Structural Optimization (BESO) based design method for lattice structure to be fabricated by additive manufacturing. Comput. Aided Des. 2015, 69, 91-101. [CrossRef]

32. Aremu, A.O.; Brennan-Craddock, J.P.J.; Panesar, A.; Ashcroft, I.A.; Hague, R.J.; Wildman, R.D.; Tuck, C. A voxel-based method of constructing and skinning conformal and functionally graded lattice structures suitable for additive manufacturing. Addit. Manuf. 2017, 13, 1-13. [CrossRef]

33. Wu, J. Continuous Optimization of Adaptive Quadtree Structures. In Proceedings of the TU Delft. Solid and Physical Modeling-SPM 2018, Bilbao, Spain, 11-13 June 2018.

34. Wu, J.; Clausen, A.; Sigmund, O. Minimum compliance topology optimization of shell-infill composites for additive manufacturing. Comput. Methods Appl. Mech. Eng. 2017, 326, 358-375. [CrossRef]

35. Wu, J.; Wang, C.C.L.; Zhang, X.; Westermann, R. Self-supporting rhombic infill structures for additive manufacturing. Comput. Aided Des. 2016, 80, 32-42. [CrossRef]

36. Tim, A.; Puentesa, J.; Kattingerb, J. Fused filament fabrication melting model. Addit. Manuf. 2018, 22, 51-59.

37. Rosenthal, M.; Henneberger, C.; Gutkes, A.; Bues, C.T. Liquid Deposition Modeling-A promising approach for 3D printing of wood. Eur. J. Wood Wood Prod. 2018, 76, 797-799. [CrossRef]

38. Heimbs, S.; Schmeer, S.; Middendorf, P.; Maier, M. Strain rate effects in phenolic composites and phenolic-impregnated honeycomb structures. Compos. Sci. Technol. 2007, 67, 2827-2837. [CrossRef]

39. Mukhopadhyay, T.; Adhikari, S. Equivalent in-plane elastic properties of irregular honeycombs: An analytical approach. Int. J. Solid Struct. 2016, 91, 169-184. [CrossRef] 